\title{
Hydrosilylation of 1-alkenes with dichlorosilane
}

\author{
Gerardus J. J. Out, Harm-Anton Klok, Leonore Schwegler, Holger Frey, \\ Martin Möller*a)
}

Department of Chemical Technology, University of Twente, P. O. Box 217, 7500 AE Enschede, The Netherlands

(Received: April 7, 1994)

\section{SUMMARY:}

Symmetrically and unsymmetrically substituted diorganodichlorosilanes have been prepared by hydrosilylation with dichlorosilane using two different platinum catalysts, i. e., hexachloroplatinic acid ("Speier's catalyst") and a platinum cyclovinylmethylsiloxane complex. Hydrosilylation of unsubstituted 1-alkenes proved to be very efficient, yielding anti-Markonikov substituted di$n$-alkyldichlorosilanes. However, no reaction was observed when electron-deficient 1 -alkenes were used. Octacarbonyldicobalt enabled formation of the monoadduct of $1 \mathrm{H}, 1 \mathrm{H}, 2 \mathrm{H}$-perfluoro-1 hexene with dichlorosilane, which was employed in a second hydrosilylation of the olefin. Thus, the anti-Markovnikov diadduct was obtained in $40 \%$ overall yield. The two-step synthesis has also been applied successfully to obtain unsymmetrically substituted diorganodichlorosilanes containing nitrile and ether groups.

\section{Introduction}

The peculiar phase behaviour displayed by poly(di- $n$-alkylsiloxane) $\mathrm{s}^{1-3}$ and poly(di-n-alkylsilylene)s ${ }^{4,5)}$ has stimulated our research on routes to introduce functional groups in the side chains of these polymers. Within this context, the synthesis is described of various diorganodichlorosilanes which can serve as monomers for the preparation of either polysiloxanes or polysilylenes.

$$
2 \mathrm{R}-\mathrm{CH}=\mathrm{CH}_{2}+\mathrm{H}_{2} \mathrm{SiCl}_{2} \stackrel{\mathrm{H}_{2} \mathrm{PtCl}_{6}}{\longrightarrow}\left(\mathrm{R}-\mathrm{CH}_{2}-\mathrm{CH}_{2}\right)_{2} \mathrm{SiCl}_{2}
$$

Although hydrosilylation reactions have been widely applied ${ }^{6-9)}$ and various mechanisms have been proposed involving homogeneous ${ }^{10}$ ) and heterogeneous catalytic species ${ }^{11-15)}$, the use of dichlorosilane in the preparation of organosilanes has been limited ${ }^{16-18}$. Dichlorosilane is a gas and should be handled with care because of its toxicity and high flammability ${ }^{19}$. However, when the reaction is performed under inert conditions in a pressure resistant reaction vessel, it presents a more efficient and versatile way to prepare diorganodichlorosilanes than organometallic reactions using Grignard reagents and tetrachlorosilane. In view of the relative lack of information on the synthetic potential of dichlorosilane, this work is intended to contribute to the existing knowledge about the hydrosilylation reaction in general.

\footnotetext{
a) Present address: Organische Chemie III, Makromolekulare Chemie, Universität Ulm, 89081 Ulm, Germany.
} 


\section{Experimental part}

\section{Materials}

Dichlorosilane $\left(\mathrm{H}_{2} \mathrm{SiCl}_{2}\right)$ (Union Carbide, 3.0) was used as received. Hexachloroplatinic acid hexahydrate $\left(\mathrm{H}_{2} \mathrm{PtCl}_{6} \cdot 6 \mathrm{H}_{2} \mathrm{O}\right)$ (Merck) was dissolved in isopropyl alcohol (Merck, p. a.) to yield a $0,05 \mathrm{~g} / \mathrm{mL}$ solution which was kept under argon. Other hydrosilylation catalysts such as platinum cyclovinylmethylsiloxane complex (PC085) (Hüls), octacarbonyldicobalt $\left(\mathrm{CO}_{2}(\mathrm{CO})_{8}\right)$ (Alfa, 98\%) and tris(triphenylphoshine)rhodium chloride $\left(\left(\mathrm{PPh}_{3}\right)_{3} \mathrm{RhCl}\right)$ (Fluka, 97\%) were used as received. 1-Butene (Hoekloos, 2.0), 1-pentene (Janssen, 97\%), 1-hexene (Aldrich, 97\%), 1-heptene (Aldrich, 97\%), 1-octene (Aldrich, 98\%), 1-nonene (Fluka, 97\%), 1-decene (Fluka, 98\%), allylbenzene (Merck, 98\%), allyl methyl ether (Alfa, 95\%), allyl ethyl ether (Janssen, 95\%), $1 H, 1 H, 2 H$-perfluoro-1-hexene (Riedel-de Haën, 99\%), $1 H, 1 H, 2 H$-perfluoro-1-decene (Riedel-de Haën, 95\%), allyl cyanide (Janssen, 98\%), allyl bromide (Aldrich, 97\%) were used without further purification.

\section{Synthesis}

Dichlorodihexylsilane (3): The symmetrical hydrosilylation of 1-hexene with dichlorosilane is described as a typical example for the preparation of di- $n$-alkyldichlorosilanes (Tab. 1).

The reaction was carried out in a stainless steel Hoke cylinder (max. pressure 300 bar). This cylinder was equipped with a stirring bar and a T-shaped stainless steel inlet, which was on one side connected to a stainless steel needle valve, whereas the other side could be closed with a screw cap. The cylinder was evacuated and flushed five times with argon. Under an argon flow, $43,7 \mathrm{~g}$ $(0,52 \mathrm{~mol}) 1$-hexene and $250 \mu \mathrm{L}$ of a $0,05 \mathrm{~g} / \mathrm{mL}$ solution of hexachloroplatinic acid in isopropyl alcohol were brought into the cylinder and the mixture was homogenized by magnetic stirring. The cyclinder was closed and cooled in liquid nitrogen, and 25,3 $\mathrm{g}(0,25 \mathrm{~mol})$ of dichlorosilane were added via a steel tube which was connected to the needle valve. The amount of dichlorosilane was checked by weighing. The reaction was allowed to proceed in a water bath $\left(15^{\circ} \mathrm{C}\right)$ under continuous stirring. Typically, the hydrosilylation was preceded by an induction period of $5-30$ $\mathrm{min}$. The onset of the reaction could be observed by a sudden temperature increase of the reaction mixture. This is in contradiction with the findings of Benkeser and Muench, who reported that dichlorosilane requires temperatures in excess of $100^{\circ} \mathrm{C}$ to react with most olefins at any convenient rate ${ }^{18)}$. Normally the mixture started to cool down after several minutes, and it was stirred for at least $2 \mathrm{~h}$ before opening of the reaction vessel. The light yellow to dark brown liquid product was removed under argon flow by means of a syringe and distilled. Yield: $49,8 \mathrm{~g} \mathrm{(74 \% )}$ of dichlorodihexylsilane (purity $98 \%$, GLC), b. p. $88-90^{\circ} \mathrm{C}(3 \mathrm{mmHg}) ; n_{\mathrm{d}}^{20}: 1,4512$ (lit. ${ }^{25)}$ : $1,4518)$.

${ }^{1} \mathrm{H}$ NMR (250 MHz): $\delta=1,60-1,16\left(\mathrm{~m}, 16 \mathrm{H},\left(\mathrm{CH}_{2}\right)_{4}\right), 1,10\left(\mathrm{t}, J=8,0 \mathrm{~Hz}, 4 \mathrm{H}, \mathrm{SiCH}_{2}\right), 0,89$ (t, $J=7,1 \mathrm{~Hz}, 6 \mathrm{H}, \mathrm{CH}_{3}$ ).

${ }^{13} \mathrm{C}$ NMR (63 MHz): $\delta=31,91$ (C3), 31,10 (C4), 22,26, 22,13 (C2, C5), 20,14 (C1), 13,85 (C6). HRMS*) $\left(\mathrm{C}_{12} \mathrm{H}_{26} \mathrm{SiCl}_{2}\right)$ : calc. 268,118 , found 268,120 .

Dibutyldichlorosilane (1): During hydrosilylation of gaseous 1-alkenes, e.g., 1-propene and 1-butene, the catalyst was brought inside the cylinder first, followed by addition of the olefin and consecutively of dichlorosilane. This procedure has also been applied to ethylene. However, when large amounts of reagents $(>100 \mathrm{~g}$ ) were used, pyrolysis occurred with concomitant expansion of the reaction vessel. Ethylene can however be hydrosilylated safely if the reaction mixture is diluted to $50 \mathrm{wt} . \%$ with an inert solvent like hexane, which had previously been distilled from potassium/benzophenone ${ }^{* *}$ ). Isolated yield; $88 \%$ (purity $96 \%$, GLC), b. p. $38-40^{\circ} \mathrm{C}(1$ $\mathrm{mmHg}$ ); $n_{\mathrm{D}}^{20}: 1,4449$ (lit.: 1,4448 ).

*) High resolution mass spectroscopy.

**) Large-scale syntheses involving allyl bromide or allyl methyl ether and dichlorosilane can also lead to hazardous side reactions and should only be performed under dilute conditions. 
${ }^{1} \mathrm{H}$ NMR (250 MHz): $\delta=1,55-1,32\left(\mathrm{~m}, 8 \mathrm{H},\left(\mathrm{CH}_{2}\right)_{2}\right), 1,10\left(\mathrm{t}, J=8,0 \mathrm{~Hz}, 4 \mathrm{H}, \mathrm{SiCH}_{2}\right), 0,92$ (t, $J=7,1 \mathrm{~Hz}, 6 \mathrm{H}, \mathrm{CH}_{3}$ ).

${ }^{13} \mathrm{C}$ NMR (63 MHz): $\delta=25,52,24,51$ (C2, C3), 20,05 (C1), 13,59 (C4).

HRMS $\left(\mathrm{C}_{8} \mathrm{H}_{18} \mathrm{SiCl}_{2}\right)$ : calc. 212,056 , found 212,056 .

Dichlorodipentylsilane (2): Isolated yield; $79 \%$ (purity $97 \%$, GLC), b. p. $70-72{ }^{\circ} \mathrm{C}(3 \mathrm{mmHg}$ ); $n_{\mathrm{D}}^{20}: 1,4489$.

${ }^{1} \mathrm{H}$ NMR (250 MHz): $\delta=1,56-1,43\left(\mathrm{~m}, 4 \mathrm{H}, \mathrm{CH}_{2} \mathrm{CH}_{2} \mathrm{CH}_{2}\right), 1,43-1,23(\mathrm{~m}, 8 \mathrm{H}$, $\left.\mathrm{CH}_{2} \mathrm{CH}_{2} \mathrm{CH}_{2}\right), 1,08\left(\mathrm{t}, J=8,1 \mathrm{~Hz}, 4 \mathrm{H}, \mathrm{SiCH}_{2}\right), 0,89\left(\mathrm{t}, J=7,0 \mathrm{~Hz}, 6 \mathrm{H}, \mathrm{CH}_{3}\right)$.

${ }^{13} \mathrm{C}$ NMR (63 MHz): $\delta=34,62(\mathrm{C} 3), 22,15,22,03$ (C2, C4), 20,27 (C1), 13,87 (C5).

HRMS $\left(\mathrm{C}_{10} \mathrm{H}_{22} \mathrm{SiCl}_{2}\right)$ : calc. 240,087 , found 240,087 .

Dichlorodiheptylsilane (4): Isolated yield; $69 \%$ (purity $97 \%$, GLC), b.p. $125-126^{\circ} \mathrm{C}$ $(5 \mathrm{mmHg}) ; n_{\mathrm{D}}^{20}: 1,4543$.

${ }^{1} \mathrm{H}$ NMR $(250 \mathrm{MHz}): \delta=1,60-1,20\left(\mathrm{~m}, 20 \mathrm{H},\left(\mathrm{CH}_{2}\right)_{5}\right), 1,10\left(\mathrm{t}, J=8,0 \mathrm{~Hz}, 4 \mathrm{H}, \mathrm{SiCH}_{2}\right), 0,90$ (t, $J=7,0 \mathrm{~Hz}, 6 \mathrm{H}, \mathrm{CH}_{3}$ ).

${ }^{13} \mathrm{C} \mathrm{NMR}$ (63 MHz): $\delta=32,42$ (C3), 31,65 (C5), 28,79 (C4), 22,64 (C6), 22,38 (C2) 20,34 (C1), $14,06(C 7)$.

HRMS $\left(\mathrm{C}_{14} \mathrm{H}_{30} \mathrm{SiCl}_{2}\right):$ calc. 296,149 , found 296,150 .

Dichlorodioctylsilane (5): Isolated yield; $63 \%$ (purity $97 \%$, GLC), b. p. $144-145^{\circ} \mathrm{C}(4 \mathrm{mmHg}$ ); $n_{\mathrm{D}}^{20}: 1,4561$.

${ }^{1} \mathrm{H}$ NMR $(250 \mathrm{MHz}): \delta=1,60-1,20\left(\mathrm{~m}, 24 \mathrm{H},\left(\mathrm{CH}_{2}\right)_{6}\right), 1,10\left(t, J=8,0 \mathrm{~Hz}, 4 \mathrm{H}, \mathrm{SiCH}_{2}\right), 0,90$ $\left(t, J=7,0 \mathrm{~Hz}, 6 \mathrm{H}, \mathrm{CH}_{3}\right)$.

${ }^{13} \mathrm{C}$ NMR (63 MHz): $\delta=32,46$ (C3), 31,85 (C6), 29,10, 29,08 (C4, C5), 22,64 (C7), 22,37 (C2) 20,34 (C1), 14,09 (C8).

HRMS $\left(\mathrm{C}_{16} \mathrm{H}_{34} \mathrm{SiCl}_{2}\right)$ : calc. 324,181 , found 324,177 .

Dichlorodinonylsilane (6): Isolated yield; $77 \%$ (purity $99 \%$, GLC), b. p. $161-162^{\circ} \mathrm{C}$ $(4 \mathrm{mmHg}) ; n_{\mathrm{D}}^{20}: 1,4575$.

${ }^{1} \mathrm{H}$ NMR (260 MHz): $\delta=1,60-1,20\left(\mathrm{~m}, 28 \mathrm{H}\left(\mathrm{CH}_{2}\right)_{7}\right), 1,10\left(t, J=8,0 \mathrm{~Hz}, 4 \mathrm{H}, \mathrm{SiCH}_{2}\right), 0,90$ (t, $J=7,0 \mathrm{~Hz}, 6 \mathrm{H}, \mathrm{CH}_{3}$ ).

${ }^{13} \mathrm{C}$ NMR (63 MHz): $\delta=32,46$ (C3), 31,87 (C7), 29,40, 29,30, 29,12 (C4-C6), 22,67 (C8), 22,38 (C2), 20,35 (C1), 14,05 (C9).

HRMS $\left(\mathrm{C}_{18} \mathrm{H}_{38} \mathrm{SiCl}_{2}\right)$ : calc. 352,212 , found 352,206 .

Dichlorodidecylsilane (7): Isolated yield; $83 \%$ (purity $95 \%$, GLC), b.p. $150-154^{\circ} \mathrm{C}$ $(0,1 \mathrm{mmHg}) ; n_{\mathrm{D}}^{20}: 1,4587$.

${ }^{1} \mathrm{H}$ NMR $(250 \mathrm{MHz}): \delta=1,60-1,20\left(\mathrm{~m}, 32 \mathrm{H},\left(\mathrm{CH}_{2}\right)_{8}\right), 1,08\left(\mathrm{t}, J=8,0 \mathrm{~Hz}, 4 \mathrm{H}, \mathrm{SiCH}_{2}\right), 0,88$ (t, $J=7,0 \mathrm{~Hz}, 6 \mathrm{H}, \mathrm{CH}_{3}$ ).

${ }^{13} \mathrm{C}$ NMR (63 MHz): $\delta=32,49$ (C3), 31,92 (C8), 29,62, 29,46, 29,33, 29,13 (C4-C7), 22,70 (C9), 22,38 (C2), 20,34 (C1), 14,12 (C10).

HRMS $\left(\mathrm{C}_{20} \mathrm{H}_{42} \mathrm{SiCl}_{2}\right)$ : calc. 380,243 , found 380,243 .

Dichloro(hexyl)hydrosilane (9): A dried and argon-flushed 300-mL Hoke cylinder was charged with $21,0 \mathrm{~g}(0,25 \mathrm{~mol}) 1$-hexene and $250 \mu \mathrm{L}$ of a $0,05 \mathrm{~g} / \mathrm{mL}$ solution of $\mathrm{H}_{2} \mathrm{PtCl}_{6} \cdot 6 \mathrm{H}_{2} \mathrm{O}$ in isopropyl alcohol. After closing, the cylinder was cooled in liquid nitrogen and $37,9 \mathrm{~g}(0,375 \mathrm{~mol})$ of dichlorosilane were condensed into the reaction mixture. The mixture was stirred for $2 \mathrm{~h}$ at room temperature, during which time only a slight amount of heat was generated. The light yellow liquid product was removed by means of a syringe and distilled. Isolated yield; $30 \mathrm{~g}(65 \%)$ (purity $\approx 95 \%$ $\left.{ }^{1} \mathrm{H} \mathrm{NMR}\right)$, b. p. $70-73^{\circ} \mathrm{C}(20 \mathrm{mmHg})$.

${ }^{1} \mathrm{H}$ NMR $(250 \mathrm{MHz}): \delta=5,50(\mathrm{t}, J=2,0 \mathrm{~Hz}, 1 \mathrm{H}, \mathrm{SiH}), 1,60-1,18\left(\mathrm{~m}, 10 \mathrm{H},\left(\mathrm{CH}_{2}\right)_{5}\right), 0,89$ (t, $J=7,1 \mathrm{~Hz}, 3 \mathrm{H}, \mathrm{CH}_{3}$ ).

${ }^{13} \mathrm{C}$ NMR (63 MHz): $\delta=31,74$ (C3), 31,30 (C4), 22,42 (C5), 21,73 (C2), 20,25 (C1), 14,02 (C6).

Butyldichlorohydrosilane (8): Isolated yield; $75 \%$ (purity $\approx 95 \%,{ }^{1} \mathrm{H}$ NMR), b.p. $120^{\circ} \mathrm{C}$ (760 $\mathrm{mmHg}$ ).

${ }^{1} \mathrm{H}$ NMR (250 MHz): $\delta=5,50(\mathrm{~s}, 1 \mathrm{H}), 1,60-1,14\left(\mathrm{~m}, 6 \mathrm{H},\left(\mathrm{CH}_{2}\right)_{3}\right), 0,92(\mathrm{t}, J=7,4 \mathrm{~Hz}, 3 \mathrm{H}$, $\mathrm{CH}_{3}$ ).

${ }^{13} \mathrm{C}$ NMR (63 MHz): $\delta=25,14,23,86(\mathrm{C} 2, \mathrm{C} 3), 19,96(\mathrm{C} 1), 13,56$ (C4). 
Dichloro(octyl)hydrosilane (10): Isolated yield; $48 \%$ (purity $\approx 95 \%,{ }^{1} \mathrm{H}$ NMR), b. p. $42{ }^{\circ} \mathrm{C}$ $(0,1 \mathrm{mmHg})$.

${ }^{1} \mathrm{H}$ NMR $(250 \mathrm{MHz}): \delta=5,50(\mathrm{~s}, 1 \mathrm{H}, \mathrm{SiH}), 1,66-1,10\left(\mathrm{~m}, 14 \mathrm{H},\left(\mathrm{CH}_{2}\right)_{7}\right), 0,90(\mathrm{t}, J=7,0$ $\left.\mathrm{Hz}, 3 \mathrm{H}, \mathrm{CH}_{3}\right)$.

${ }^{13} \mathrm{C}$ NMR (63 MHz): $\delta=32,11$ (C3), 31,84 (C6), 29,09 (C4, C5), 22,65 (C7), 21,79 (C2), 20,29 (C1), 14,05 (C8).

Dichloro(decyl)hydrosilane (11): Isolated yield; $83 \%$ (purity $\approx 95 \%,{ }^{1} \mathrm{H}$ NMR), b. p. $85-88^{\circ} \mathrm{C}$ $(2-3 \mathrm{mmHg})$.

${ }^{1} \mathrm{H}$ NMR $(250 \mathrm{MHz}): \delta=5,50(\mathrm{~s}, 1 \mathrm{H}, \mathrm{SiH}), 1,66-1,16\left(\mathrm{~m}, 18 \mathrm{H},\left(\mathrm{CH}_{2}\right)_{9}\right), 0,90(\mathrm{t}, J=7,0$ $\left.\mathrm{Hz}, 3 \mathrm{H}, \mathrm{CH}_{3}\right)$.

${ }^{13} \mathrm{C}$ NMR (63 MHz): $\delta=32,08(\mathrm{C} 3), 31,90(\mathrm{C} 8), 29,57,29,40,29,31,29,12(\mathrm{C} 4-\mathrm{C} 7), 22,68$ (C9), 21,78 (C2), 20,26 (C1), 14,10 (C10).

Dichloro/3-(2-ethoxyethoxy)propylJhydrosilane (12): Isolated yield; $25 \%$ (purity $\approx 95 \%,{ }^{1} \mathrm{H}$ NMR), b. p. $48-50^{\circ} \mathrm{C}(0,1 \mathrm{mmHg})$.

${ }^{1} \mathrm{H}$ NMR $(250 \mathrm{MHz}): \delta=5,50(\mathrm{~s}, 1 \mathrm{H}, \mathrm{SiH}), 3,52\left(\mathrm{~s}, 4 \mathrm{H},-\mathrm{OCH}_{2} \mathrm{CH}_{2} \mathrm{O}-\right), 3,52-3,40(\mathrm{~m}$, $4 \mathrm{H}, \mathrm{CH}_{3} \mathrm{CH}_{2} \mathrm{O}-$ and $-\mathrm{OCH}_{2} \mathrm{CH}_{2}-$ ), 1,78 (quintet, $J=6,5 \mathrm{~Hz}, 2 \mathrm{H},-\mathrm{CH}_{2} \mathrm{CH}_{2} \mathrm{Si}-$ ) 1,20 (t, $\left.J=7,8 \mathrm{~Hz}, 2 \mathrm{H},-\mathrm{CH}_{2} \mathrm{C}_{2} \mathrm{Si}-\right), 1,15\left(\mathrm{t}, J=5,9 \mathrm{~Hz}, 3 \mathrm{H}, \mathrm{CH}_{3}\right)$.

${ }^{13} \mathrm{C}$ NMR (63 MHz): $\delta=71,65(\mathrm{C} 3), 70,15,69,62(\mathrm{C} 4, \mathrm{C} 5), 66,54(\mathrm{C} 6), 22,63(\mathrm{C} 2), 17,81(\mathrm{C} 1)$, 15,08 (C7).

Dichloro( $(H, 1 H, 2 H, 2 H$-perfluorohexyl)hydrosilane (13): A dried and argon-flushed Hoke $500-\mathrm{mL}$ cylinder was charged with $4,48 \mathrm{~g}$ of $\mathrm{Co}_{2}(\mathrm{CO})_{8}$ and $125,2 \mathrm{~g}(0,509 \mathrm{~mol})$ of $1 \mathrm{H}, 1 \mathrm{H}, 2 \mathrm{H}$-perfluorohexene. After cooling in liquid nitrogen, $22,3 \mathrm{~g}(0,221 \mathrm{~mol})$ of dichlorosilane were condensed into the reaction mixture. The cyclinder was closed and the mixture was allowed to stir overnight at room temperature. No heat evolution could be observed. Distillation of the crude product yielded $37,9 \mathrm{~g}(49,4 \%)$ of 13 (purity $\approx 95 \%$, ${ }^{1} \mathrm{H} \mathrm{NMR}$ ), b. p. $55^{\circ} \mathrm{C}(16 \mathrm{mmHg})$, together with $60 \mathrm{~g}$ of unreacted olefin and $17,1 \mathrm{~g}$ of an unidentified yellow oil, b.p. $94-95^{\circ} \mathrm{C}$ (3 $\mathrm{mmHg})$.

${ }^{1} \mathrm{H}$ NMR $(250 \mathrm{MHz}): \delta=5,61(\mathrm{~s}, 1 \mathrm{H}, \mathrm{SiH}), 2,30\left(\mathrm{~m}, 2 \mathrm{H},-\mathrm{CF}_{2} \mathrm{CH}_{2}-\right), 1,47(\mathrm{t}, J=8,4 \mathrm{~Hz}$, $\left.2 \mathrm{H},-\mathrm{CH}_{2} \mathrm{Si}\right)$.

${ }^{13} \mathrm{C}$ NMR $(63 \mathrm{MHz}): \delta=119,78,117,76,115,19,(\mathrm{C} 3-\mathrm{C} 5), 24,23\left(\mathrm{t}, J_{\mathrm{C}-\mathrm{CF}}=23,7 \mathrm{~Hz}, \mathrm{C} 2\right)$, $10,73(\mathrm{C} 1)$.

Dichloro(3-ethoxypropyl)hexylsilane (15): The synthesis of 15 serves as an example for the hydrosilylation of allyl derivatives with monoalkyldichlorosilanes. A $100-\mathrm{mL}$ three-neck flask equipped with a reflux condenser, stirring bar and septum was charged with 50,2 $\mathrm{g}(0,271 \mathrm{~mol})$ of dichloro(hexyl)hydrosilane. With a syringe $23,3 \mathrm{~g}(0,271 \mathrm{~mol})$ of allyl ethyl ether was added through the septum. After homogenization, this was followed by addition of $560 \mu \mathrm{L}$ of a 0,05 $\mathrm{g} / \mathrm{mL}$ solution of $\mathrm{H}_{2} \mathrm{PtCl}_{6} \cdot 6 \mathrm{H}_{2} \mathrm{O}$ in isopropyl alcohol. The mixture was stirred at r.t. for 120 h. Distillation yielded $41,6 \mathrm{~g}(57 \%)$ of 15 (purity $91 \%$, GLC), b. p. $108-112^{\circ} \mathrm{C}(1 \mathrm{mmHg}) ; n_{\mathrm{D}}^{20}$ : 1,4495 .

${ }^{1} \mathrm{H}$ NMR (250 MHz): $\delta=3,47\left(\mathrm{q}, J=7,1 \mathrm{~Hz}, 2 \mathrm{H}, \mathrm{CH}_{3} \mathrm{CH}_{2} \mathrm{O}-\right.$ ), $3,42(\mathrm{t}, J=6,4 \mathrm{~Hz}, 2 \mathrm{H}$, $\left.-\mathrm{OCH}_{2} \mathrm{CH}_{2}-\right), 1,88-1,6\left(\mathrm{~m}, 2 \mathrm{H},-\mathrm{OCH}_{2} \mathrm{CH}_{2}-\right), 1,60-1,05\left(\mathrm{~m}, 15 \mathrm{H}, \mathrm{CH}_{3} \mathrm{CH}_{2} \mathrm{O}-\right.$ and $\left.-\mathrm{CH}_{2} \mathrm{Si}\left(\mathrm{CH}_{2}\right)_{5}-\right), 0,89\left(\mathrm{t}, \mathrm{J}=7,5 \mathrm{~Hz}, 3 \mathrm{H}, \mathrm{CH}_{3}\right)$.

${ }^{13} \mathrm{C}$ NMR (63 MHz): $\delta=71,78(\mathrm{C} 3), 66,12\left(\mathrm{C}^{\prime}\right), 32,12(\mathrm{C} 3), 31,30(\mathrm{C} 4), 22,82-22,30(\mathrm{C} 2$, $\left.\mathrm{C} 5, \mathrm{C}^{\prime}\right), 20,32(\mathrm{C} 1), 16,92\left(\mathrm{C1}^{\prime}\right), 15,16\left(\mathrm{C5}^{\prime}\right), 14,05(\mathrm{C} 6)$.

MS: $m / z=237(0,4), 235\left(1, \mathrm{M}^{+}-\mathrm{Cl}\right), 187(38), 185(67), 157(47), 145(22), 143(41), 115$ (24), 99 (9), 59 (100), 31 (25).

Butyldichloro(3-cyanopropyl)silane (14): Isolated yield; $72 \%$ (purity $96 \%, \mathrm{GLC}$ ), (b. p. $100^{\circ} \mathrm{C}$ (3 $\mathrm{mmHg}) ; n_{\mathrm{D}}^{20}: 1,4633$.

${ }^{1} \mathrm{H}$ NMR (250 MHz): $\delta=2,43\left(\mathrm{t}, J=6,9 \mathrm{~Hz}, 2 \mathrm{H},-\mathrm{CH}_{2} \mathrm{CN}\right.$ ), 1,87 (quintet, $J=7,7 \mathrm{~Hz}, 2 \mathrm{H}$, $\left.\left.\mathrm{CH}_{2} \mathrm{CH}_{2} \mathrm{CH}_{2} \mathrm{CN}\right)\right), 1,50-1,33\left(\mathrm{~m}, 4 \mathrm{H},-\mathrm{CH}_{2} \mathrm{CH}_{3}\right) 1,22$ (t, $J=8,4 \mathrm{~Hz}, 2 \mathrm{H}$, $\left.\mathrm{CNCH}_{2} \mathrm{CH}_{2} \mathrm{CH}_{2} \mathrm{Si}-\right), 1,12\left(\mathrm{t}, J=7,9 \mathrm{~Hz}, 2 \mathrm{H}, \mathrm{SiCH}_{2} \mathrm{C}_{3} \mathrm{H}_{7}\right), 0,90\left(\mathrm{t}, J=7,1 \mathrm{~Hz}, 3 \mathrm{H}, \mathrm{CH}_{3}\right.$ ).

${ }^{13} \mathrm{C}$ NMR (63 MHz): $\delta=118,82(\mathrm{CN}), 25,26,24,18(\mathrm{C} 2, \mathrm{C} 3), 19,79,19,57,19,13,18,95(\mathrm{C} 1$, $\left.\mathrm{C}^{\prime}, \mathrm{C}^{\prime}, \mathrm{C}^{\prime}\right), 13,39$ (C4).

MS: $m / z=224(0,2), 222\left(0,2, \mathrm{M}^{+}-1\right), 170(10), 168(82), 166(100), 155(14), 138(33), 127$ (6), $113(16), 99(10), 63$ (9), 41 (6). 
Dichloro[3-(2-ethoxyethoxy)propyl]hexylsilane (16): Isolated yield; $26 \%$ (purity $\approx 90 \%,{ }^{1} \mathrm{H}$ $\mathrm{NMR})$, b. p. $98-100^{\circ} \mathrm{C}(0,05 \mathrm{mmHg})$.

${ }^{1} \mathrm{H}$ NMR (250 MHz): $\delta=3,58\left(\mathrm{~s}, 4 \mathrm{H},-\mathrm{OCH}_{2} \mathrm{CH}_{2} \mathrm{O}-\right), 3,55-3,45\left(\mathrm{~m}, 4 \mathrm{H}, \mathrm{CH}_{3} \mathrm{CH}_{2} \mathrm{O}-\right.$ and $\left.-\mathrm{OCH}_{2} \mathrm{CH}_{2}-\right), 1,85-1,72\left(\mathrm{~m}, 2 \mathrm{H},-\mathrm{OCH}_{2} \mathrm{CH}_{2}\right), 1,60-1,05\left(\mathrm{~m}, 15 \mathrm{H}, \mathrm{CH}_{2} \mathrm{CH}_{2} \mathrm{O}-\right.$ and $\left.-\mathrm{CH}_{2} \mathrm{Si}\left(\mathrm{CH}_{2}\right)_{5}-\right), 0,89\left(\mathrm{t}, J=7,5 \mathrm{~Hz}, 3 \mathrm{H}, \mathrm{CH}_{3}\right)$.

${ }^{13} \mathrm{C}$ NMR (63 MHz): $\delta=72,47\left(\mathrm{C}^{\prime}\right), 70,19,69,85\left(\mathrm{C5}^{\prime}, \mathrm{C}^{\prime}\right), 66,65\left(\mathrm{C} 6^{\prime}\right), 32,14(\mathrm{C} 3), 31,33$ (C4), 22,71, 22,47, 22,34 (C2, C5, C2'), 20,33 (C1), 16,82 (C1'), 15,17 (C7'), 14,06 (C6).

Dichloro[3-(2-ethoxyethoxy)propyl]octylsilane (17): Isolated yield; $40 \%$ (purity $\approx 90 \%,{ }^{1} \mathrm{H}$ $\mathrm{NMR})$, b. p. $138^{\circ} \mathrm{C}(0,05 \mathrm{mmHg})$.

${ }^{1} \mathrm{H}$ NMR (250 MHz): $\delta=3,55\left(\mathrm{~s}, 4 \mathrm{H},-\mathrm{OCH}_{2} \mathrm{CH}_{2} \mathrm{O}-\right), 3,52-3,38\left(\mathrm{~m}, 4 \mathrm{H}, \mathrm{CH}_{3} \mathrm{CH}_{2} \mathrm{O}-\right.$ and $\left.-\mathrm{OCH}_{2} \mathrm{CH}_{2}-\right), 1,85-1,72\left(\mathrm{~m}, 2 \mathrm{H},-\mathrm{OCH}_{2} \mathrm{CH}_{2}-\right), 1,55-1,00\left(\mathrm{~m}, 19 \mathrm{H}, \mathrm{CH}_{3} \mathrm{CH}_{2} \mathrm{O}-\right.$ and $\left.-\mathrm{CH}_{2} \mathrm{Si}\left(\mathrm{CH}_{2}\right)_{7}-\right), 0,85\left(\mathrm{~m}, 3 \mathrm{H}, \mathrm{CH}_{3}\right)$.

${ }^{13} \mathrm{C}$ NMR (63 MHz): $\delta=72,46(\mathrm{C} 3), 70,10,69,76\left(\mathrm{C}^{\prime}, \mathrm{C} 5^{\prime}\right), 66,60\left(\mathrm{C} 6^{\prime}\right), 32,41(\mathrm{C} 3), 31,80$ (C6), 29,05, 29,02 (C4, C5) 22,63, 22,58, 22,30 (C2, C7, C2'), 20,26 (C1), 16,74 (C1'), 15,09 (C7'), 14,03 (C8).

Dichloro(hexyl)-IH,1H,2H,2H-perfluorohexylsilane (18): A 100 -mL three-neck flask equipped with a reflux condenser, stirring bar and septum was charged with $30 \mathrm{~g}(0,161 \mathrm{~mol})$ of dihloro(hexyl)hydrosilane. With a syringe $40,6 \mathrm{~g}(0,165 \mathrm{~mol})$ of $1 H, 1 H, 2 H$-perfluorohexene was added through the septum. Under vigorous stirring, $350 \mu \mathrm{L}$ of platinum cyclovinylmethylsiloxane catalyst (PC085, Hüls) was added. The clear transparent mixture turned from light yellow to brown with concomitant generation of heat. The mixture was allowed to stir overnight at room temperature. Distillation yielded $48,1 \mathrm{~g}(69 \%)$ of 18 (purity $97 \%$, GLC), b. p. $97-100^{\circ} \mathrm{C}(4-5 \mathrm{mmHg}) ; n_{\mathrm{D}}^{20}: 1,3860$.

${ }^{1} \mathrm{H}$ NMR (250 MHz): $\delta=2,27\left(\mathrm{tt}, J_{\mathrm{H}-\mathrm{F}}=17,3 \mathrm{~Hz}, J_{\mathrm{H}-\mathrm{H}}=8,6,2 \mathrm{H}, \mathrm{CF}_{2} \mathrm{CH}_{2} \mathrm{CH}_{2} \mathrm{Si}\right)$, $1,65-1,22\left(\mathrm{~m}, 10 \mathrm{H}, \mathrm{CF}_{2} \mathrm{CH}_{2} \mathrm{CH}_{2} \mathrm{Si}\right.$ and $\left.\left(\mathrm{CH}_{2}\right)_{4}\right), 1,15\left(\mathrm{t}, J=7,9 \mathrm{~Hz}, 2 \mathrm{H}, \mathrm{SiCH}_{2}\right), 0,88(\mathrm{t}, J=$ $\left.7,5 \mathrm{~Hz}, 3 \mathrm{H}, \mathrm{CH}_{3}\right)$.

${ }^{13} \mathrm{C}$ NMR (63 MHz): $\delta=119-110\left(\mathrm{C6}^{\prime}-\mathrm{C} 33^{\prime}\right), 32,03(\mathrm{C} 3), 31,26(\mathrm{C} 4), 24,68\left(\mathrm{t}, J_{\mathrm{C}-\mathrm{CF}_{2}}=\right.$ $23,5 \mathrm{~Hz}, \mathrm{C2}^{\prime}$ ), 22,44 (C5), 22,17 (C2), 20,09 (C1), 13,98 (C6), 10,30 (C1').

MS: $m / z=346(1), 344\left(2, \mathrm{M}^{+}-\mathrm{C}_{6} \mathrm{H}_{14}\right), 209(47), 189(49), 185$ (16), 183 (26) $169(13), 145$ (49), 141 (32), 139 (23), 119 (18), 77 (13), 57 (16), 43 (100).

Dichloro(decyl)-lH,1H,2H,2H-perfluorodecylsilane (19): Isolated yield; $66 \%$ (purity $92 \%$, GLC), b. p. $144-146^{\circ} \mathrm{C}(0,1 \mathrm{mmHg}) ; n_{\mathrm{D}}^{20}: 1,3831$.

${ }^{1} \mathrm{H}$ NMR (250 MHz): $\delta=2,27\left(\mathrm{tt}, J_{\mathrm{H}-\mathrm{F}}=17,3 \mathrm{~Hz}, J_{\mathrm{H}-\mathrm{H}}=8,6,2 \mathrm{H}, \mathrm{CF}_{2} \mathrm{CH}_{2} \mathrm{CH}_{2} \mathrm{Si}\right)$, $1,63-1,22\left(\mathrm{~m}, 18 \mathrm{H}, \mathrm{CF}_{2} \mathrm{CH}_{2} \mathrm{CH}_{2} \mathrm{Si}\right.$ and $\left.\left(\mathrm{CH}_{2}\right)_{8}\right), 1,15\left(\mathrm{t}, J=7,9 \mathrm{~Hz}, 2 \mathrm{H}, \mathrm{CH}_{2} \mathrm{Si}\right), 0,88(\mathrm{t}, J=$ $\left.7,5 \mathrm{~Hz}, 3 \mathrm{H}, \mathrm{CH}_{3}\right)$.

${ }^{13} \mathrm{C}$ NMR (63 MHz): $\delta=32,37$ (C3), 31,91 (C8), 29,59, 29,42, 29,32, 29,08 (C4-C7), 24,76 (t $\left.\left(J_{\mathrm{C}-\mathrm{CF}}=23,5 \mathrm{~Hz}\right), \mathrm{C} 2^{\prime}\right), 22,69(\mathrm{C} 9), 22,21(\mathrm{C} 2), 20,09(\mathrm{C} 1), 14,06(\mathrm{C} 10), 10,32\left(\mathrm{C} 1^{\prime}\right)$.

MS: $m / z 546(2), 544\left(4, \mathrm{M}^{+}-\mathrm{C}_{10} \mathrm{H}_{22}\right), 409(99), 363(22), 345$ (45), 339 (100), $295(35), 241$ (27), $239(40), 169(27), 141$ (18), $119(27), 85$ (72), 71 (62), 57 (53), 43 (26).

Bis $(I H, 1 H, 2 H, 2 H$-perfluorohexyl)dichlorosilane (20): A dried and argon-flushed $100-\mathrm{mL}$ three-neck flask equipped with condenser, stirring bar and septum was charged with $28,3 \mathrm{~g}(0,115$ mol) $1 H, 1 H, 2 H$-perfluorohexene. Via the septum $0,5 \mathrm{~mL}$ of a $3 \%$ platinum cyclovinylmethylsiloxane complex (PC085, Hüls) was added, followed by addition of $37,9 \mathrm{~g}(0,109 \mathrm{~mol})$ of 13 . Immediately after addition of the silane, the yellow colour of the reaction mixture disappeared and then changed to brown, which was accompanied by significant heat evolution. The reaction mixture was allowed to stir overnight at room temperature. Distillation yielded $56,1 \mathrm{~g}(86 \%)$ of a colourless oil, b. p. $83-84{ }^{\circ} \mathrm{C}\left(3 \mathrm{mmHg}\right.$ ) (purity $\approx 90 \%,{ }^{1} \mathrm{H} \mathrm{NMR}$; due to decomposition on the column, the purity could not be checked by GLC); $n_{\mathrm{D}}^{20}: 1,3448$.

${ }^{1} \mathrm{H}$ NMR (250 MHz): $\delta=2,30\left(\mathrm{~m}, 4 \mathrm{H},-\mathrm{CF}_{2} \mathrm{CH}_{2}-\right), 1,42\left(\mathrm{t}, J=8,5 \mathrm{~Hz}, 4 \mathrm{H}, \mathrm{CH}_{2} \mathrm{Si}\right.$ ).

${ }^{13} \mathrm{C}$ NMR (63 MHz): $\delta=119,82,117,72,115,24$ (C3-C6), 24,69 (t $\left.(J=23,6 \mathrm{~Hz}), \mathrm{C} 2\right), 10,49$ (C1).

Bis(3-ethoxypropyl)dichlorosilane $(21)^{5)}$ : Isolated yield; $15 \%$ (purity $\approx 95 \%,{ }^{1} \mathrm{H}$ NMR), b.p. $123-124^{\circ} \mathrm{C}(4 \mathrm{mmHg})$.

${ }^{1} \mathrm{H}$ NMR (250 MHz): $\delta=3,44\left(\mathrm{q}, J=7,1 \mathrm{~Hz}, 4 \mathrm{H}, \mathrm{CH}_{3} \underline{\mathrm{CH}}_{2} \mathrm{O}\right), 3,39(\mathrm{t}, J=6,4 \mathrm{~Hz}, 4 \mathrm{H}$, $\left.\mathrm{CH}_{2} \mathrm{CH}_{2} \mathrm{O}\right), 1,80-1,68\left(\mathrm{~m}, 4 \mathrm{H}, \mathrm{CH}_{2}\right), 1,16\left(\mathrm{t}, J=7,0 \mathrm{~Hz}, 6 \mathrm{H}, \mathrm{CH}_{3}\right), 1,14(\mathrm{t}, J=8,3 \mathrm{~Hz}, 4 \mathrm{H}$, $\mathrm{SiCH}_{2}$ ). 
${ }^{13} \mathrm{C}$ NMR (63 MHz): $\delta=71,63$ (C3), 66,03 (C4), 22,76 (C2), 16,89 (C1), 15,08 (C5).

MS: $m / z=237(2), 237\left(6, \mathrm{M}^{+}-\mathrm{Cl}\right), 209(14), 207(36), 189(7), 187(53), 185(80), 159(33)$, $157(53), 145$ (27), $143(42), 115(36), 99(6), 59$ (100), 41 (14).

Bis[3-(2-ethoxyethoxy)propyl]dichlorosilane (22): Isolated yield; $25 \%$ (purity $\approx 95 \%,{ }^{1} \mathrm{H}$ NMR), b. p. $115^{\circ} \mathrm{C}(0,05 \mathrm{mmHg})$.

${ }^{1} \mathrm{H}$ NMR (250 MHz): $\delta=3,50\left(\mathrm{~s}, 8 \mathrm{H},-\mathrm{OCH}_{2} \mathrm{CH}_{2} \mathrm{O}-\right), 3,48-3,32\left(\mathrm{~m}, 8 \mathrm{H}, \mathrm{CH}_{3} \mathrm{CH}_{2} \mathrm{O}-\right.$ and $\left.\mathrm{OCH}_{2} \mathrm{CH}_{2}-\right), 1,78-1,60\left(\mathrm{~m}, 4 \mathrm{H},-\mathrm{CH}_{2} \mathrm{CH}_{2} \mathrm{Si}-\right), 1,12\left(\mathrm{t}, J=7,4 \mathrm{~Hz}, 6 \mathrm{H}, \mathrm{CH}_{3}\right)$, $1,12-1,05\left(\mathrm{~m}, 4 \mathrm{H},-\mathrm{CH}_{2} \mathrm{CH}_{2} \mathrm{Si}-\right)$.

${ }^{13} \mathrm{C} \mathrm{NMR}(63 \mathrm{MHz}): \delta=72,35$ (C3), 70,06, 70,00 (C4, C5), 66,53 (C6), 22,56 (C2), 16,67 (C1), $15,07(\mathrm{C} 7)$.

Bis(5-hexenyl)dichlorosilane (23): Isolated yield: $38 \%$ (purity $90 \%$ ), b. p. $90-10^{\circ} \mathrm{C}(1 \mathrm{mmHg}$ ); $n_{\mathrm{D}}^{20} ; 1,4698$.

${ }^{1} \mathrm{H}$ NMR (250 MHz): $\delta=5,90-5,70\left(\mathrm{~m}, 2 \mathrm{H}, \mathrm{CH}=\mathrm{CH}_{2}\right), 5,60-5,25(\mathrm{~m}, 1 \mathrm{H}), 5,10-4,90(\mathrm{~m}$, $\left.4 \mathrm{H}, \mathrm{CH}=\mathrm{CH}_{2}\right), 2,15-2,00\left(\mathrm{~m}, 4 \mathrm{H}, \mathrm{CH}_{2} \mathrm{CH}=\right), 1,70-1,38\left(\mathrm{~m}, 8 \mathrm{H}, \mathrm{CH}_{2} \mathrm{CH}_{2}\right), 1,15-1,00(\mathrm{~m}$, $4 \mathrm{H}, \mathrm{SiCH}_{2}$ ). (C1).

${ }^{13} \mathrm{C}$ NMR (63 MHz): $\delta=138,39,114,69$ (C5, C6), 33,21, 31,59 (C3, C4), 21,84 (C2), 20,16

Bis(3-phenylpropyl)dichlorosilane (24): Isolated yield; 63\% (purity 95\%, GLC), b.p. $188-192{ }^{\circ} \mathrm{C}(0,1 \mathrm{mmHg})$.

${ }^{1} \mathrm{H}$ NMR (250 MHz): $\delta=7,62-7,16\left(\mathrm{~m}, 10 \mathrm{H}, \mathrm{C}_{6} \mathrm{H}_{5}\right), 2,69\left(\mathrm{t}, J=7,5 \mathrm{~Hz}, 4 \mathrm{H}, \mathrm{C}_{6} \mathrm{H}_{5} \mathrm{CH}_{2}\right.$ ), 1,83 (quintet, $J=7,9 \mathrm{~Hz}, 4 \mathrm{H}, \mathrm{CH}_{2} \mathrm{CH}_{2} \mathrm{CH}_{2}$ ), $1,11\left(\mathrm{t}, J=8,4 \mathrm{~Hz}, 4 \mathrm{H}, \mathrm{SiCH}_{2}\right.$ ).

${ }^{13} \mathrm{C}$ NMR (63 MHz): $\delta=141,32,128,47-128,39,126,01$ (C4-C9), 38,35, 24,15 (C2, C3), $19,77(\mathrm{C} 1)$.

HRMS $\left(\mathrm{C}_{18} \mathrm{H}_{22} \mathrm{SiCl}_{2}\right)$ : Calc. 336,087, found 336,085.

\section{Methods}

Analytical gas-liquid chromatography (GLC) was performed on a Varian 3400 instrument equipped with a DS 654 data station. A DB-5 capillary column was used $(30 \mathrm{~m} \times 0,32 \mathrm{~mm} \times$ $0,25 \mu \mathrm{m}$ ) in combination with a flame ionization detector (FID). Refractive indices were determined at $20^{\circ} \mathrm{C}$ using a Carl-Zeiss Abbé refractometer. Mass spectra (HRMS) were measured on a Finnigan MAT 90 spectrometer $(70 \mathrm{eV})$. The mass spectral data are listed as follows: $\mathrm{m} / \mathrm{z}$ (relative intensity). Solution ${ }^{1} \mathrm{H}$ and proton-decoupled ${ }^{13} \mathrm{C}$ NMR spectra were recorded on a Bruker AC 250 spectrometer at $250,3 \mathrm{MHz}$ and $62,9 \mathrm{MHz}$, respectively. $\mathrm{CDCl}_{3}$ was used as a solvent and tetramethylsilane (TMS) as a reference. $\mathrm{C} 1$ refers to the carbon atom attached to silicon. Assignments of ${ }^{13} \mathrm{C}$ resonances are based on $2 \mathrm{D}$ correlation experiments reported before on $n$-alkyl-substituted polysilylenes ${ }^{26)}$.

\section{Results and discussion}

From a series of small scale hydrosilylations carried out in Carius tubes, the following observations can be summarized:

A peculiar feature of the hydrosilylation reaction is the induction period, which precedes the reaction, irrespective of the reactivity of the reagents. It has been postulated that during this time, the platinum catalyst is reduced by hydrosilane compounds to yield $\operatorname{Pt}(0)$ colloids, which are the actually active species ${ }^{11-15)}$. However, in our hands, induction periods were also observed when a $\operatorname{Pt}(0)$ catalyst such as platinum cyclovinylmethylsiloxane complex was directly added to the reaction mixture. In fact, both Speier's catalyst and the Pt( 0$)$ reagent showed comparable activity in hydrosilylation reactions with dichlorosilane and monoorganodichlorosilanes, with regard to efficiency as well as regioselectivity. 
The regioselectivity of the hydrosilylation of 1 -alkenes is reported to be about $95 \%$ anti-Markovnikov ${ }^{6-9}$. This is confirmed by the results described in this chapter. Tab. 1 lists the different dichlorosilanes which have been prepared. Generally, it was observed by means of GLC and ${ }^{13} \mathrm{C}$ NMR that the crude product contained approximately 4-5\% Markovnikov products, which could be removed by repeated distillation using a spinning band distillation column.

Although the hydrosilylation of 1-alkenes with dichlorosilane constituted an efficient method for the preparation of di- $n$-alkyldichlorosilanes, it was observed that the reaction of dichlorosilane with functionalized 1-alkenes was frequently hindered by side reactions. In many cases, these were accompanied by precipitation of the catalyst or evolution of hydrogen. In case of electron-deficient 1 -alkenes like $1 \mathrm{H}, 1 \mathrm{H}, 2 \mathrm{H}$-perfluoroolefins and allyl cyanide, monoorganodichlorosilanes appeared to react smoothly and in high yields, whereas with dichlorosilane no reaction occurred. In fact, several unsuccessful attempts have been made to react dichlorosilane with $1 \mathrm{H}, 1 \mathrm{H}, 2 \mathrm{H}$-perfluorohexene, e.g., in the presence of Speier's catalyst, platinum cyclovinylmethylsiloxane complex and tris(triphenylphoshine)rhodium(I) chloride (Wilkinson's catalyst). The cobaltcarbonyl complex $\mathrm{Co}_{2}(\mathrm{CO})_{8}$ appeared to be the only catalyst which enabled the hydrosilylation of $1 \mathrm{H}, 1 \mathrm{H}, 2 \mathrm{H}$-perfluorohexene with dichlorosilane, contradicting earlier data which claim that only electron-rich olefins can be hydrosilylated with this reagent ${ }^{20}$.

Allyl ethers, which have frequently been used as reagents in hydrosilylation reactions, e. g., in polymer-analogous modification reactions of polymethylhydrosiloxane ${ }^{21,22)}$, appeared to be much less efficient when dichlorosilane was used as the silylating reagent. In case of allyl methyl ether, allyl ethyl ether and allyl 2-ethoxyethyl ether, reactions had to be activated by stirring the mixture for at least $30 \mathrm{~min}$ at $50^{\circ} \mathrm{C}$. The symmetrical hydrosilylation reaction was complicated by side reactions, and generally low conversions and concomitantly low yields $(10-20 \%)$ were obtained. Precipitation of black material might indicate that these effects are due to poisoning of the catalyst ${ }^{23,24)}$. Slightly better yields $(20-30 \%)$ were possible when the reaction was allowed to proceed in two steps, first by reacting an excess of dichlorosilane with the allyl ether, followed by purification of the monoadduct, which served as starting compound for the second hydrosilylation step.

Hydrosilylation of 1,5-hexadiene was performed in a 7:1 molar excess of the diene relative to dichlorosilane, to avoid side reactions, e.g., polymerization. Nevertheless, extensive rearrangement of the double bond occurred, which resulted in the formation of a large number of isomers.

\section{Conclusions}

The platinum-catalyzed hydrosilylation of 1-alkenes with dichlorosilane presents an efficient and versatile way to prepare diorganodichlorosilanes with anti-Markovnikov configuration. The synthesis of dichlorosilanes bearing functional groups, such as ether and nitrile as well as perfluoroalkyl moieties, is also feasible. In general, Speier's catalyst and platinum cyclovinylmethylsiloxane complex resemble each other with respect to reactivity and regioselectivity. In case of electron-deficient 1-alkenes, 


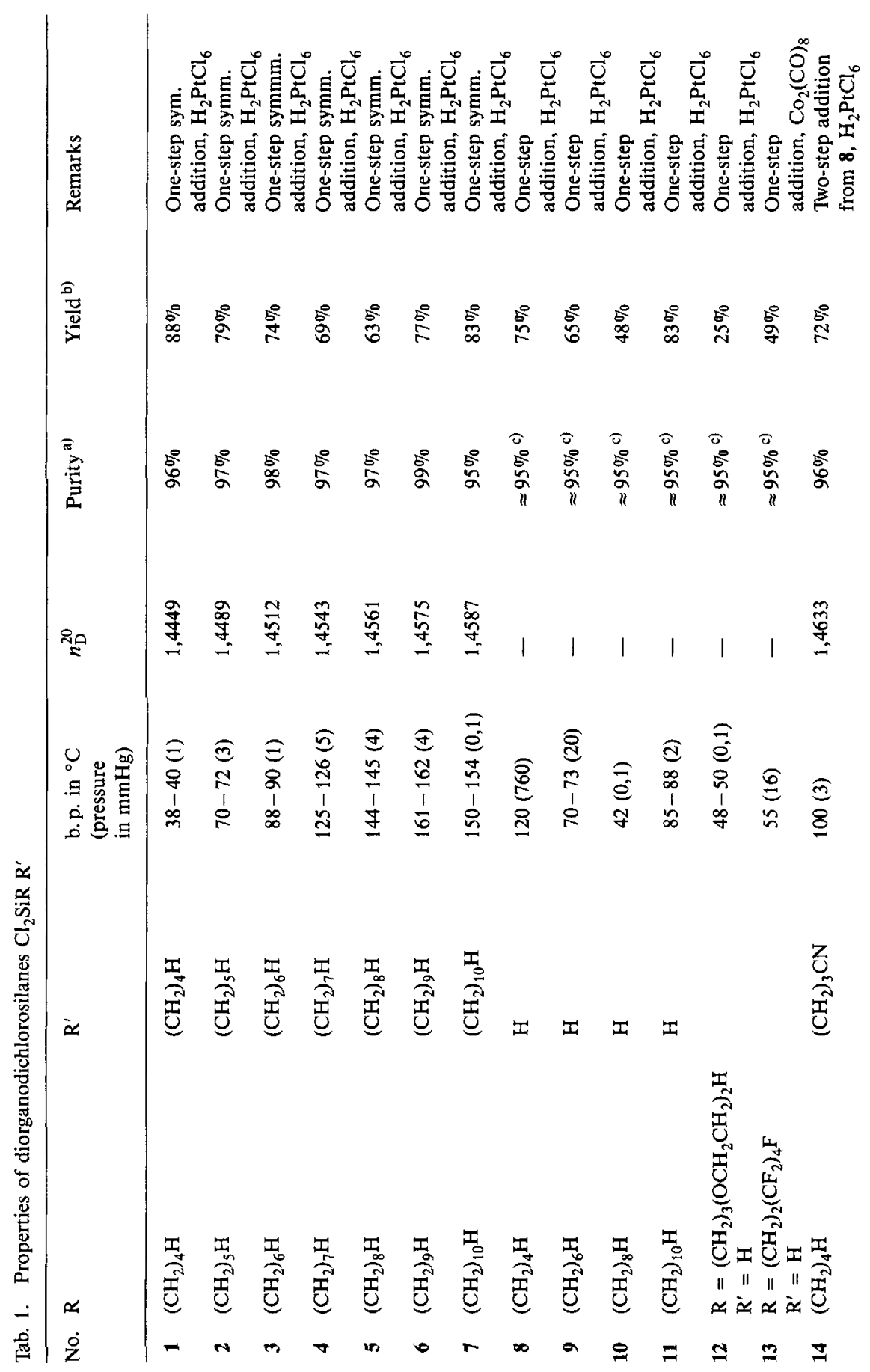




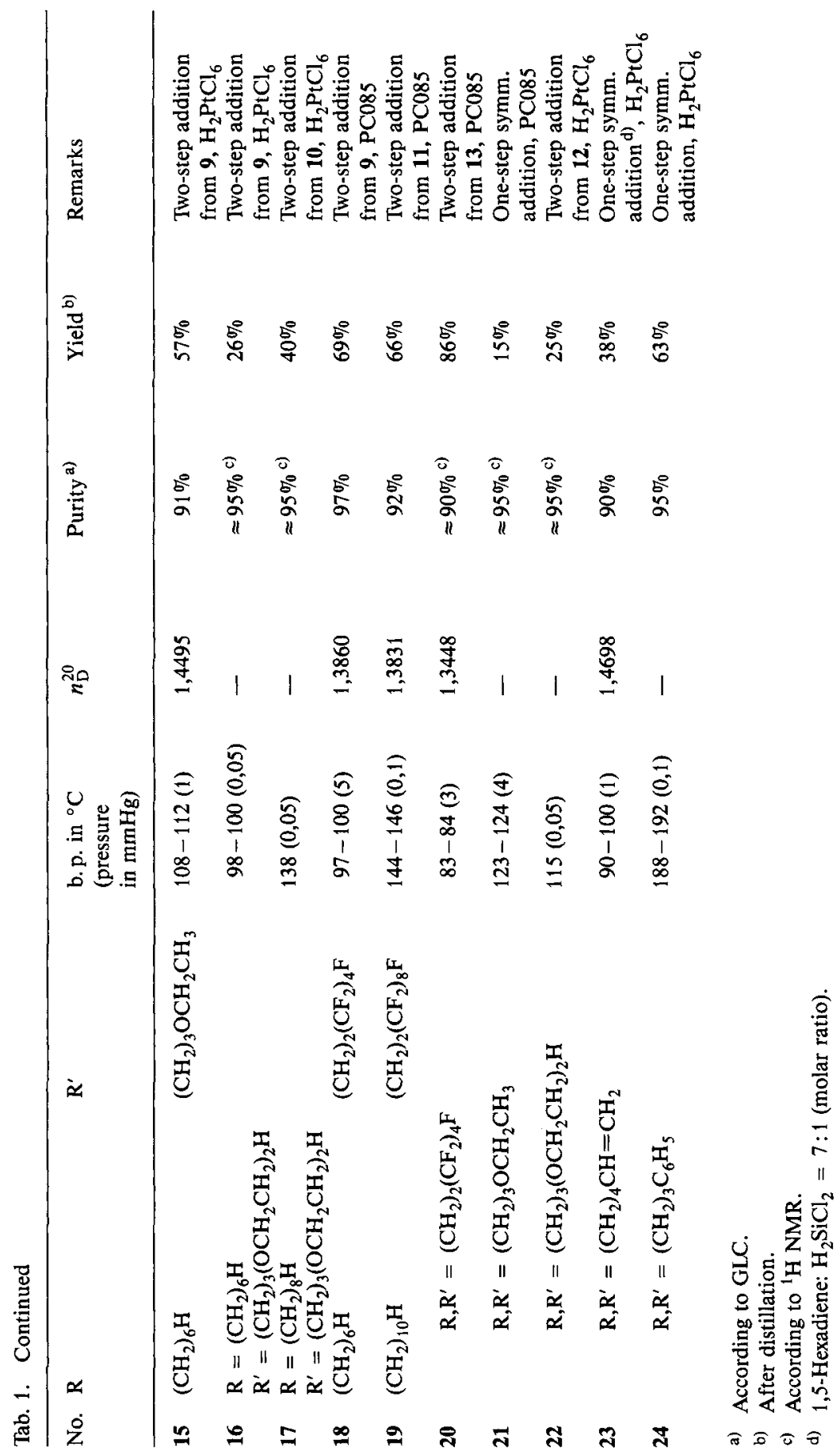


monoorganodichlorosilanes appeared to be more reactive silylating reagents than dichlorosilane. Use of the cobaltcarbonyl complex $\mathrm{Co}_{2}(\mathrm{CO})_{8}$ enabled hydrosilylation of $1 H, 1 H, 2 H$-perfluorohexene with dichlorosilane.

Summarizing, dichlorosilane constitutes a useful reagent in the synthesis of diorganodichlorosilanes, yielding new synthetic pathways for the preparation of functionalized polysiloxanes and polysilylenes.

The authors like to thank Dr. L. Wilczek of Du Pont Experimental Station for his suggestions regarding the hydrosilylation of fluorinated alkenes.

1) G. Kögler, K. Loufakis, M. Möller, Polymer 31, 1538 (1990)

2) M. Möller, S. Siffrin, G. Kögler, D. Oelfin, Makromol. Chem., Macromol. Symp. 34, 171 (1990)

3) G. J. J. Out, A. Turetskii, M. Möller, D. Oelfin, Macromolecules, in press (1994)

4) H. Frey, K. Matyjaszewski, M. Möller, D. Oelfin, Colloid Polym. Sci. 269, 442 (1991)

5) H. Frey, G. J. J. Out, M. Möller, D. Greszta, K. Matyjaszewski, Macromolecules 26, 6231 (1993)

6) J. L. Speier, Adv. Organomet. Chem. 17, 407 (1979)

7) I. Ojima, in: "The Chemistry of Organofunctional Silicon Compounds", S. Patai, Z. Rappoport, Eds., Wiley, New York 1989, Chapter 25, p. 1479

8) B. Marciniec, J. Gulinski, W. Urbaniak, Z. W. Kornetka, "Comprehensive Handbook on Hydrosilylation", B. Marciniec, Ed., Pergamon, Oxford 1992

9) B. Marciniec, J. Gulinski, J. Organomet. Chem. 15, 446 (1993)

10) A. J. Chalk, J. F. Harrod, J. Am. Chem. Soc. 87, 16, 1133, 1640 (1965)

11) L. N. Lewis, N. Lewis, J. Am. Chem. Soc. 108, 7228 (1986)

12) L. N. Lewis, N. Lewis, Chem. Mater. 1, 106 (1989)

13) L. N. Lewis, R. J. Uriarte, Organometallics 9, 621 (1990)

14) L. N. Lewis, R. J. Uriarte, N. Lewis, J. Mol. Catal. 66, 105 (1991)

15) L. N. Lewis, R. J. Uriarte, N. Lewis, J. Catal. 67, 127 (1991)

16) R. A. Benkeser, D. F. Ehler, J. Organomet. Chem. 69, 193 (1974)

17) R. A. Benkeser, E. C. Mozdzen, W. C. Muench, R. T. Roche, M. P. Siklosi, J. Org. Chem. 44, 1370 (1979)

18) R. A. Benkeser, W. C. Muench, J. Organomet. Chem. C3-C9, 184 (1980)

19) K. G. Sharp, A. Arvidson, T. C. Elvey, J. Electrochem. Soc. 129, 2346 (1982)

${ }^{20)}$ G. K.-I. Magomedov, J. Organomet. Chem. 385, 113 (1990)

21) A. M. Garnault, "Nouveaux silicones et polystyrènes fluorés: Préparation et Caractérisation"; Thèse, Université Pierre et Marie Curie, Paris 1986

${ }^{22)}$ R. Dorigo, "Synthèse et caractérisation physico-chimique de polysiloxannes fluorés", Thèse, Université Pierre et Marie Curie, Paris 1989

23) A. Molnár, J. T. Kiss, I. Bucsi, T. Katona, M. Bartok, J. Mol. Catal. 61, 307 (1990)

24) A. Onopchenko, E. T. Sabourin, J. Org. Chem. 52, 4118 (1987)

25) P. G. Kaatz, G. D. Patterson, H. K. Kim, H. Frey, K. Matyjaszewski, Mater. Res. Soc. Symp. Proc. (1991); 214 (Opt. Electr. Prop. Polym.) 17-22

26) F. C. Schilling, F. A. Bovey, J. M. Zeigler, Macromolecules 19, 2309 (1986) 